Two-year progression-free-survival Two-year overall-survival
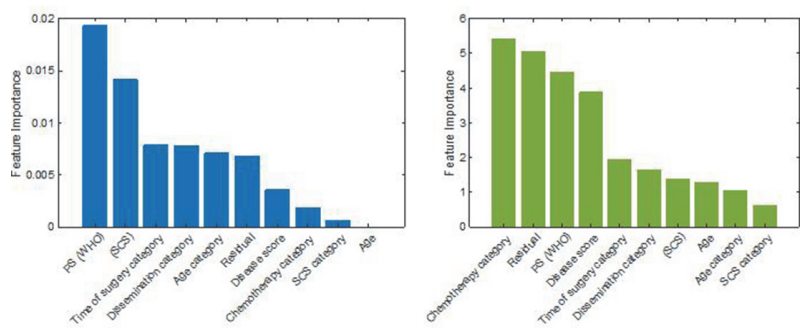

Abstract 282 Figure 1

prognosis estimation was formulated as a binary classification problem. Dataset was split into training (80\%) and test (20\%) cohorts with repeated random sampling until there was no significant difference $(p=0.20)$ between the two cohorts. A ten-fold cross-validation was applied. Various state-of-the-art supervised ML classifiers were tested, including Support-Vector-Machines (SVMs), K-Nearest Neighbors (KNNs), Ensemble Classifiers, and Naïve Bayes, based on a set of performance metrics. These results were directly compared to conventional Logistic Regression (LR). For feature selection, multivariate feature ranking using the MRMR method was carried out.

Result(s)* Two hundred nine patients were identified. The model's mean prediction accuracy reached $73 \%$. We demonstrated that SVM and Ensemble Discriminant algorithms outperformed Logistic Regression in accuracy indices. The probability of achieving a cancer-free state was maximized with a combination of primary cytoreduction, good performance status, and maximal surgical effort (AUC 0.63). Standard chemotherapy, performance status, tumor load, and residual disease were consistently predictive of the two-year overall survival (AUC 0.63-0.66) (figure 1). The model recall and precision were greater than $80 \%$.

Conclusion* Appropriate feature selection is required when building a HGSOC model for two-year prognosis prediction. For HGSOC prognosis, one should consider not only the patient's disease burden but also their overall medical status and ability to undergo extensive surgery, resulting in survival benefits alongside with standard chemotherapy.

\section{ANAPLASTIC MURAL NODULES WITHIN MUCINOUS OVARIAN CARCINOMA, A CASE SERIES ASSESSING TREATMENTS AND OUTCOMES}

L Baxter*, RK Ali Mohan, C Ayres, P Cohen, Y Leung. King Edward Memorial Hospital, Subiaco, Australia

\subsection{6/ijgc-2021-ESGO.377}

Introduction/Background* Mucinous ovarian tumours account for $\sim 10 \%$ of primary ovarian neoplasia and a generally diagnosed at an early stage with relatively favourable oncological outcomes. However those possessing anaplastic carcinoma within mural nodules rarer still and carry with them a poor prognosis and lack of consensus regarding their optimal treatment regime. Whilst a great deal of research has been published focusing on their histopathological and immunohistochemical characteristics, there is limited evidence nor consensus regarding their ideal adjuvant treatment regimes. This study sought to combine cases from our unit with a meta-analysis of cases in the literature to provide insight into current treatment regimes and outcomes.

Methodology A systematic review was conducted of the English language literature to identify articles published regarding outcomes and treatment modalities of patients having anaplastic carcinoma foci within mucinous ovarian tumours. References of these articles were reviewed to identify all possible cases in the literature. Where treatment regimes were not listed in the publications the contact author was reached for comment. These cases were then combined with 7 cases from our own institution for a multivariate and survival analysis.

Result(s)* A total of 66 cases were identified in the literature. Average age 43.7 (median 40.5), range 15-74yo. 83\% of patients underwent a total abdominal hysterectomy, $17 \%$ of patients did not under go a hysterectomy, with the remaining 2 cases having been done laparoscopically. $70 \%$ of patients underwent a BSO, the remainder a USO. $50 \%$ of cases were FIGO stage IA1 at the time of diagnosis, of these $16 \%$ died during follow up with 3 of those dying within 12 months of diagnosis.

Conclusion* Anaplastic mural nodules arising on a background of mucinous ovarian carcinoma are associated with heterogenous outcomes when considering progression free survival and overall survival. Their treatment within the literature is highly variable, particularly regarding adjuvant therapy. Patients with improved overall survival and progression free survival were more likely to be lower stage and have a smaller adnexal mass at diagnosis.

\section{CAN HE4 REPLACE CA 125 AS A BIOMARKER IN OVARIAN CANCER?}

A Ranjan, H Dubey, P Tanwar. AllMS, Delhi, Lab Oncology, Delhi, India

\subsection{6/ijgc-2021-ESG0.378}

Introduction/Background* Ovarian cancer is currently diagnosed using CAl25, which has a number of fallacies. Recently HE4 (human epididymis protein) is an emerging biomarker. It has higher potency to differentiate benign tumours from malignant one.

Methodology We studied 123 cases of ovarian cancer confirmed by histopathological examination. Whole blood samples were collected at the time of diagnosis prior to therapy (chemotherapy or surgery). We tested them for serum level of CA 125 \& HE4. Cut off values for HE4 and CA125 were <57.6 $\mathrm{pmol} / \mathrm{L}$ and $<39.6 \mathrm{U} / \mathrm{mL}$ respectively. Cut off values were calculated by ROC Curve analysis.

Result(s)* Total 123 cases were evaluated for serum level of HE4 \& CA125 prior to therapy. Results are being displayed in table 1 .

Out of 123 cases 38 showed CA 125 values negative, whereas the same was 14 for HE4, indicating a better diagnostic performance by HE4. As studied by Drapkin et al 2005, serum level of HE4 will not be raised in 50\% cases of clear-cell variant and almost all cases of mucinous tumors. It is positive in 93\% cases of serous tumors and almost all cases of endometrioid tumors. It will not be raised in benign ovarian cysts.

Among premenopausal cases (42/123), 10 showed CA 125 negative where as HE4 was negative in $7 / 42$ cases. Out of 10 CA 125 negative cases, 5 were HE4 positive. Out of HE4 
Abstract 287 Table 1 Overian cancer cases $(n=123)$ showing negativetive equivocal and positive result for CA 125 \& HE4

\begin{tabular}{|l|l|l|l|l|l|l|}
\hline CA 123 & HE4 \\
\hline Negative (N) & $\begin{array}{l}\text { Equivocal } \\
\text { (E) }\end{array}$ & Positive (P) & N & E & P \\
\hline \multicolumn{7}{|l|}{ All ovarian cancer cases (pre + post menopausal; n= 123) } \\
\hline 38 & 18 & 67 & 14 & 63 & 45 \\
\hline Premenopausal cases (42/123) & 6 & 26 & 7 & 21 & 14 \\
\hline 10 & 6 & 45 & 7 & 41 & 33 \\
\hline Postmenopausal cases (81/123) & 11 & &
\end{tabular}

negative cases 2/7 showed CA 125 positive result. Mucinous and clear cell variants show HE4 negative result.

Among postmenopausal cases (81/123), 25 showed CA 125 negative where as HE4 was negative in 7/42 cases. 18/25 CA 125 negative cases showed positive HE4 results. None of HE4 negative case showed higher CA 125 value.
Conclusion* Study shows HE is more accurate in diagnosing OC \& differentiating it from benign tumors. The study is continued to achieve a decisive conclusion.

\section{FREQUENCY OF PATHOGENIC MUTATIONS AND PROGNOSTIC IMPACT OF GERMLINE GENE PANEL TESTING IN PATIENTS WITH PRIMARY EPITHELIAL OVARIAN CANCER}

${ }^{1,2}$ B Ataseven*, ${ }^{1} \mathrm{P}$ Harter, ${ }^{3} \mathrm{~K}$ Rhiem, ${ }^{1,4} \mathrm{~F}$ Heitz, ${ }^{1} \mathrm{~S}$ Schneider, ${ }^{1} \mathrm{M}$ Bommert, ${ }^{1} \mathrm{~A}$ Traut, ${ }^{3}$ RK Schmutzler, ${ }^{1} \mathrm{~A}$ Du Bois. 'Evang. Huyssens-Stiftung Essen-Huttrop (eine Einrichtung der KEM | Evang. Kliniken Essen-Mitte gGmbH), Gynaecology and Gynaecologic Oncology, Essen, Germany; '2University Hospital, LMU Munich, Department of Obstetrics and Gynecology; ${ }^{3}$ University Hospital Cologne, Medical Faculty, , Center for Familial Breast and Ovarian Cancer, Center for Integrated Oncology (CIO), , Köln, Germany; ${ }^{4}$ Universitätsmedizin Berlin, corporate member of Freie Universität Berlin, HumboldtUniversität zu Berlin, and Berlin Institute of Health, Berlin, Department for Gynecology with the Center for Oncologic Surgery Charité Campus Virchow-Klinikum, Berlin

10.1136/ijgc-2021-ESGO.379

Abstract 291 Table 1 Univariate and multivariate analysis of prognostic factors for overall survival (OS)

\begin{tabular}{|c|c|c|c|c|c|c|c|}
\hline \multirow[t]{2}{*}{ os } & \multirow[t]{2}{*}{ Total } & \multirow[t]{2}{*}{ Events } & \multirow[t]{2}{*}{ Median OS } & \multicolumn{2}{|c|}{ Univariate } & \multicolumn{2}{|c|}{ Multivariate } \\
\hline & & & & HR (CI95\%) & $P$ & HR (Cl95\%) & $P$ \\
\hline$n$ & 569 & 140 & 55 & & & & \\
\hline \multicolumn{8}{|l|}{ Type of surgery } \\
\hline PDS & $413(72.6)$ & $93(22.5)$ & 61 & 1 (ref.) & & 1 & \\
\hline IDS & $156(27.4)$ & $47(30.1)$ & 40 & $2.09(1.45-3.00)$ & $<0.001$ & $2.79(1.86-4.19)$ & $<0.001$ \\
\hline \multicolumn{8}{|c|}{$\begin{array}{l}\text { ECOG performance } \\
\text { status }\end{array}$} \\
\hline 0 & $543(95.4)$ & $133(25.4)$ & 55 & 1 & & 1 & \\
\hline$\geq 1$ & $26(4.6)$ & $7(26.9)$ & 25 & $1.96(0.91-4.20)$ & 0.084 & $1.35(0.59-3.12)$ & 0.474 \\
\hline \multicolumn{8}{|l|}{ Albumin (g/L) } \\
\hline$\geq 35$ & $470(82.6)$ & $112(23.8)$ & 55 & 1 & & 1 & \\
\hline$<35$ & $46(8.1)$ & $16(34.8)$ & 44 & $1.68(0.99-2.84)$ & 0.053 & $1.25(0.70-2.23)$ & 0.449 \\
\hline unknown & $53(9.3)$ & $12(22.6)$ & 71 & & & $0.50(0.26-0.95)$ & 0.035 \\
\hline \multicolumn{8}{|l|}{$\mathrm{ACCl}$} \\
\hline $0-1$ & $248(43.6)$ & $59(23.8)$ & 60 & 1 & & 1 & \\
\hline $2-3$ & $248(43.6)$ & $60(24.2)$ & 55 & $1.18(0.83-1.70)$ & 0.358 & $1.11(0.76-1.60)$ & 0.589 \\
\hline$\geq 4$ & $73(12.8)$ & $21(28.8)$ & 36 & $1.79(1.09 .2-95)$ & 0.022 & $1.58(0.96-2.62)$ & 0.074 \\
\hline \multicolumn{8}{|l|}{ Ascites (mL) } \\
\hline$\leq 500$ & $431(75.7)$ & $93(21.6)$ & 62 & 1 & & 1 & \\
\hline$>500$ & $138(24.3)$ & $47(34.1)$ & 43 & $1.90(1.34-2.71)$ & $<0.001$ & $1.75(1.14-2.68)$ & 0.010 \\
\hline \multicolumn{8}{|c|}{ History of previous } \\
\hline no cancer & $492(86.5)$ & $121(24.6)$ & 55 & 1 & & 1 & \\
\hline breast cancer & $41(7.2)$ & $8(19.5)$ & 76 & $0.77(0.38-1.57)$ & 0.474 & $0.74(0.34-1.62)$ & 0.454 \\
\hline $\begin{array}{l}\text { other type of } \\
\text { cancer }\end{array}$ & $36(6.3)$ & $11(30.6)$ & 39 & $1.49(0.80-2.76)$ & 0.211 & $1.39(0.70-2.75)$ & 0.340 \\
\hline \multicolumn{8}{|c|}{$\begin{array}{l}\text { Residual disease } \\
\text { after surgery }(\mathrm{mm})\end{array}$} \\
\hline RDO & $422(74.2)$ & $80(19)$ & 71 & 1 & & 1 & \\
\hline $\mathrm{RD} \geq 1$ & $147(25.8)$ & $60(40.8)$ & 36 & $2.81(2.01-3.94)$ & $<0.001$ & $2.87(1.99-4.14)$ & $<0.001$ \\
\hline \multicolumn{8}{|l|}{ FIGO } \\
\hline FIGO III & $261(45.9)$ & $48(18.4)$ & 76 & 1 & & 1 & \\
\hline FIGO IV & $308(54.1)$ & $92(29.9)$ & 47 & $1.79(1.26-2.55)$ & 0.001 & $1.27(0.87-1.26)$ & 0.217 \\
\hline \multicolumn{8}{|l|}{ Germline result } \\
\hline No mutation & $430(75.6)$ & $115(26.7)$ & 47 & 1 & & 1 & \\
\hline BRCA1/2mut & $108(19)$ & $20(18.5)$ & - & $0.49(0.30-0.78)$ & 0.003 & $0.49(0.31-0.80)$ & 0.004 \\
\hline Other mutation & $31(5.4)$ & $5(16.1)$ & 71 & $0.44(0.18-1.08)$ & 0.074 & $0.34(0.14-0.85)$ & 0.021 \\
\hline
\end{tabular}

